\title{
Determination of Capillary Motion of Water in Bricks Using Neutron Radiography
}

\author{
A. Czachor, AbD El-Ghany El-AbD and J.J. MilczareK \\ Institute of Atomic Energy, Świerk, 05-400 Otwock
}

\begin{abstract}
Vertical migration of water in several porous materials has been directly observed and recorded with the recently installed neutron and gamma radiography facility at the MARIA reactor of Institute of Atomic Energy, Świerk. The density profiles of water in samples were obtained from the optical density of digitised images. A simple model (representing the porous material as the collection of capillary tubes of various radii) has been introduced to describe the liquid transport in porous materials in terms of capillary motion. The equation for viscous movement of water in porous material in presence of the gravitation is proposed. A good agreement of the calculated density profiles for vertical water migration with the data collected for siliceous bricks suggests that the thinnest capillary tubes determine the upper edge of the water density profile.
\end{abstract}

PACS numbers: $81.05 . \mathrm{Rm}, 28.20 .-\mathrm{v}, 47.55 . \mathrm{Mh}$

\section{Introduction}

There are several methods of determination of the moisture content inside porous materials. Destructive methods - gravimetric and carbide apparatus are most commonly used [1]. They are time-consuming and poor in the spatial resolution of the moisture distribution; besides the sampling involves laborious slicing or strike boring, which interfere with the liquid flow process. Also the non-destructive methods based on the electrical resistance or dielectric constant measurements give only a rough assessment of the moisture content and no information on moisture distribution. Other non-destructive methods are: the nuclear magnetic resonance (NMR) [2] which is based on the interaction of electromagnetic radiation with the liquid's hydrogen, and the radiography methods which 
are based on attenuation of radiation passing through the porous materials, using neutrons [3], gamma and X-rays [4]. Detailed references to can be found in monographs and reviews of the field - see e.g. [5].

The neutron radiography is a well-known non-destructive method capable for visualisation and analysis of moisture distribution in materials partially transparent for neutrons, like sandstone or building materials [6, 7]. Its effectiveness stems from the very strong scattering of neutrons by hydrogen atoms and allows for a direct measurement of the hydrogenous liquid density profile in a porous medium. This method should be perhaps favoured to the other methods, because it is fast, quantitative, of high sensitivity and good spatial resolution. Using it, the time dependent transport and distribution of water in bricks have been studied here in real time.

\section{Experiment}

The water distribution study in bricks was performed with the recently completed neutron and gamma radiography (NRG) station at the nuclear reactor MARIA of the Institute of Atomic Energy (IAE) at Świerk [8]. The experiments were performed at the characteristic rate $L / d=150$, where $d$ is the neutron-collimator-aperture diameter and $L$ is the aperture-sample distance. Slightly conical divergent neutron beam $\left(10^{7} \mathrm{n} / \mathrm{cm}^{2} \mathrm{~s}\right.$ at the sample position) was directed to the sample and after absorption and scattering on the components of the sample the transmitted neutrons reached the fluorescent screen, which converts the neutron radiation to visible light (Fig. 1). The light is directed by a

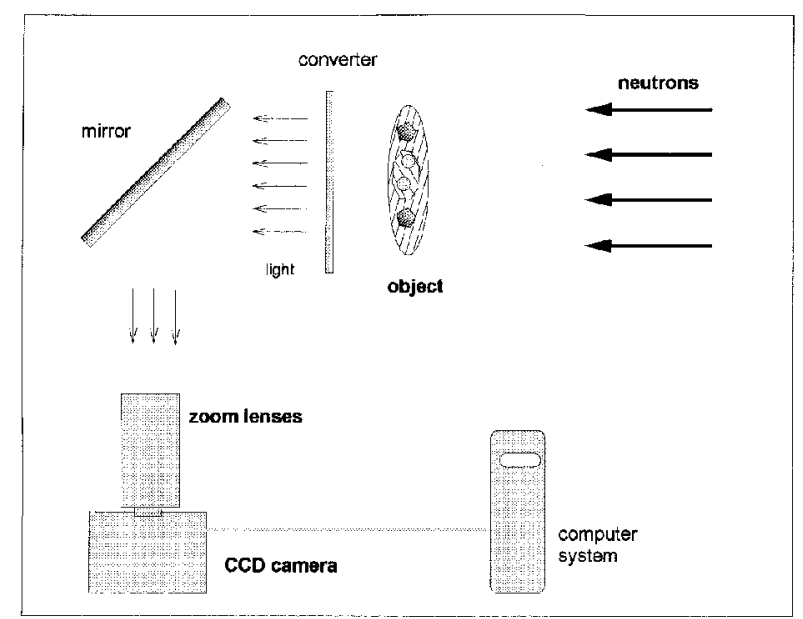

Fig. 1. Scheme of the Neutron and Gamma Radiography station at the nuclear reactor MARIA at Świerk. 
high-quality mirror to the lenses of the CCD camera of high sensitivity. The Hamamatsu ORCA-ER monochrome digital camera was used and two-processor work station served as the picture acquisition and camera control system.

A rectangular prism specimen $(160 \times 42 \times 20 \mathrm{~mm})$ of siliceous brick was initially coated from all sides, except lower end face, with the three layers of paint. Then it was placed vertically with its lower end supported by four pins in the container. The water was allowed into container and the recording started after the water reached the fixed level of $5 \mathrm{~mm}$ from the lower end of the sample. The distance between the neutron converter screen and the brick side was $40 \mathrm{~mm}$. The images were recorded every $10 \mathrm{~min}$. On the recorded images the upper light region of the image represents the dry part of the brick and can be easily distinguished from the darker lower region representing the wet part. The movement of the upper edge of dark grey region marks the water ascent in the sample (Fig. 2).

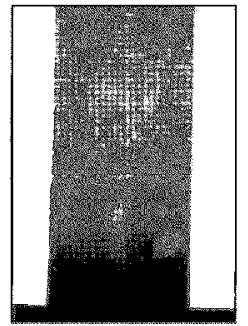

$4936 \mathrm{~s}$

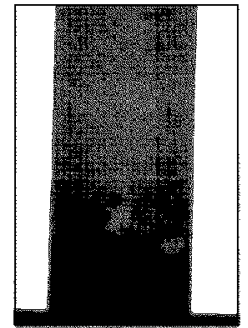

$10080 \mathrm{~s}$

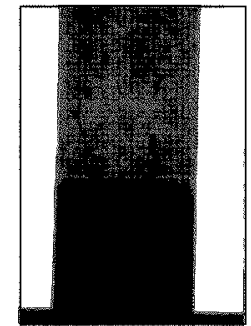

282005

Fig. 2. Neutron radiography patterns of water in silicate brick at three subsequent times of the water rise from the bottom. The brick is not structurally homogeneous see lighter spots in the patterns.

The optical density profiles were taken along the fixed vertical line going across the centre of the brick's image. The water density profiles were obtained from those profiles by subtracting the background optical density determined for the dry brick.

\section{Model}

Theoretical analysis of the liquid distributions and movements in porous material is based mainly on the application of Darcy equation to unsaturated flow theory developed in soil physics [10], recently applied also to building materials [11]. The liquid transport inside the material can be then described by a generalised nonlinear diffusion equation with the diffusion parameter $D$ depending on the liquid content. This equation can be written in several forms to serve different experimental conditions. Solutions of this diffusion equation were mainly numerical and lots of different methods were developed to solve it, depending on the initial and the boundary conditions [10]. 
One often introduces the model of porous material consisting of capillary tubes. In its simplest form it can represent the one-dimensional horizontal flow in a capillary tubes neglecting gravity, or the vertical capillary rise against gravity (the infiltration equation). As the forces acting on liquid in the capillary tubes are approximately known, it is possible to describe many aspects of such transport using Newtonian mechanics. The forces are: the gravity force pulling the liquid down, the Young-Laplace-Reyleigh capillary force which can drive it along the tube, and the Poiseuille damping force due to the interaction of the liquid with the tube walls (viscosity). However, due to the damping the acceleration term in the Newton equation is usually assumed negligible [5].

In this paper we aim at a semi-quantitative description of liquid movement in a porous medium, understood to be a collection of cylindrical capillary tubes of various radii, in terms of the radius distribution function $\rho(r)$, such that $\int \rho(r) \mathrm{d} r=1$. To be able to do such calculation analytically, we depart from the above mentioned classic theories and develop a simpler intuitive approach. We assume that the liquid in a capillary tube is pulled up by the capillary force $F_{u}$, proportional to the circumference of the tube's cross-section, i.e. $F_{u}=\alpha r$ (where $r$ is the radius of the tube and $\alpha$ is a constant), and pulled down by the gravitation with the force $F_{d}=-g c \pi r^{2} h$, where $h$ is the height of water in the tube, $c$ is the liquid density and $g=9.81 \mathrm{~m} / \mathrm{s}^{2}$. At equilibrium the sum of these forces $F$ is zero and one obtains the maximum height $h_{0}=\alpha /(\pi \mathrm{cgr})$ of the liquid in the tube.

Now, as the water movement is strongly damped, due to viscosity, we assume here the "Archimedean law" to be approximately valid: velocity is proportional to force. It gives the equation

$$
\mathrm{d} h(t) / \mathrm{d} t=\gamma F=\gamma r(\alpha-\pi c g r h),
$$

where $\gamma$ is a proportionality constant. On assuming $h(0)=0$ at time $t=0$, one obtains the solution

$$
h(t, r)=p[1-\exp (-k r)] / r,
$$

where $p=\alpha /(\pi c g), k=\alpha \gamma t$. Using it we can easily determine the velocity of the liquid in its tube

$$
v(r, t)=\mathrm{d} h / \mathrm{d} t=v_{0} \exp (-A t),
$$

where $v_{0}=\alpha \gamma^{2} /(\pi c g)$ does not depend on $r$, while $A=\alpha \gamma r$. Clearly, due to the linear $r$-dependence of $A$, the vertical transport of liquid in narrow capillary tubes is faster than in the broad ones. These formulae offer a good description of the liquid behaviour in capillary tubes at the time $t>1 / A$. Let us note the characteristic time dependence of the water front movement: from the linear rise at small $t$ (or $k$ ) to a levelling off at the height $p / r_{1}$. The well-known proportionality (see e.g. [9]) of the water front height to $t^{1 / 2}$ at small times is not shown here.

Let the $z$-axis of the coordinate system be directed vertically. Using the well-known step function $\theta(z)$ we can represent the liquid content in a tube by the 
number 1 in the liquid range, 0 above, as $\theta[h(r, t)-z]$. With the radius distribution function $\rho(r)$ of the tubes the mass of the liquid in the tubes attributed to the $\mathrm{d} z$ range is $\pi c r^{2} \rho(r) \theta(h(r, t)-z) \mathrm{d} z \mathrm{~d} r$. On integrating with respect to $r$ we obtain for the liquid mass density along the $z$-axis the following expression:

$$
m(z, t)=\pi c \int_{h(r, t)>z} \rho(r) r^{2} \mathrm{~d} r .
$$

To arrive at more convenient range of integration we have to invert the function $h(r, t)$ so that $r$ is given in terms of $t$ and $z$. This cannot be done analytically with the function derived above. However, as we are at this moment interested mainly in a semi quantitative extraction of $\rho(r)$ from experimental data, let us use now, instead of the $h(r, t)$, a more convenient function of a similar concave shape, $h(r, t) \rightarrow p k /(1+k r)$, having the same limits at $r=0$ and $r \rightarrow \infty$. This function can be easily inverted, and we have a convenient formulation of the mass profile along the $z$-axis at time $t$ (or $k$

$$
m(z, k)=\pi c \int_{0}^{p / z-1 / k} \rho(r) r^{2} \mathrm{~d} r .
$$

Physically one should expect the tube radii to be not too small and not too large. As a very instructive example let us take the truncated $\rho(r)$, proportional to $r^{s-2}$ for $r_{1}<r<r_{2}$, zero otherwise, $s$ being a constant to be estimated. We obtain (neglecting constant factors)

$$
m(z, k)= \begin{cases}{\left[r_{2}^{s+1}-r_{1}^{s+1}\right] /(s+1),} & p / z-1 / k>r_{2} \\ {\left[(p / z-1 / k)^{s+1}-r_{1}^{s+1}\right] /(s+1),} & r_{1}<p / z-1 / k<r_{2} \\ 0, & p / z-1 / k<r_{1} .\end{cases}
$$

Typical plots of this function are shown in Fig. 3 for $s=-5 / 4$.

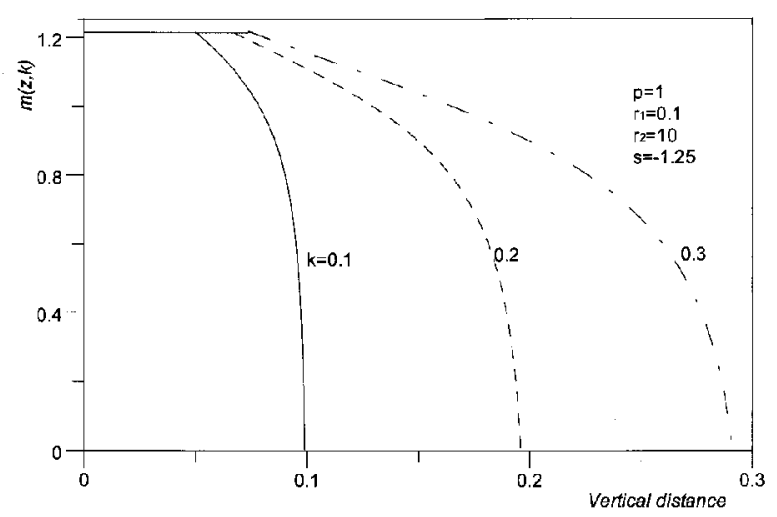

Fig. 3. Typical water profiles following from Eq. (7), calculated for three times represented by the parameter $k$ (arbitrary units). 
There are always two special points in such plots. The kink at small $z_{1}=$ $k p /\left(k r_{2}+1\right)$ is related to the upper bound of the $\rho(r)$ at $r_{2}$ - the water front in the thick tubes is coming late. The water front in fast tubes appears at the larger $z_{2}=k p /\left(k r_{1}+1\right)$, being attributed to the smallest tube radius $r_{1}$. Some model liquid profiles for different times of vertical flow are shown in Fig. 3.

\section{Discussion}

After subtracting the dry brick level of neutron signal we have arrived at the experimental water density profile in material along the vertical dimension of the sample, see Fig. 4. These data show a lot of scatter, due to the inhomogeneous structure of this material, so one can try to extract from them only rough characteristics of the corresponding radii distribution function $\rho(r)$. Qualitatively,

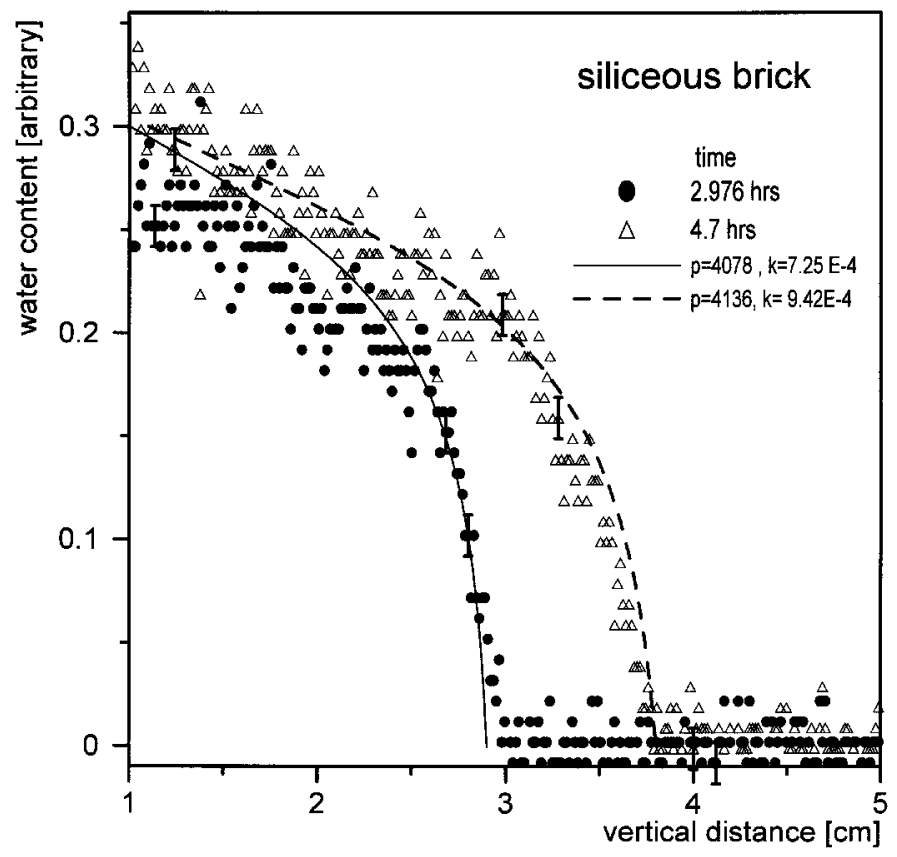

Fig. 4. Water density distribution $m(z, t)$ in the brick shown in Fig. 2, vertical scan, raw data after subtracting the dry brick intensities. Open triangles and black circles the data taken after 3 and 4.7 hours, respectively. Lines - the model fits, corresponding to the input data $z_{1}=1 \mathrm{~cm}, z_{2}=2.9 \mathrm{~cm}$ for open dots and $z_{1}=1.1 \mathrm{~cm}, z_{2}=3.8 \mathrm{~cm}$ for black circles. The output data, assuming $m(0, t)=0.3$ (in arbitrary units), $s=-5 / 4$, $r_{2}=100 r_{1}$, are $k=7.3, p=4.1$ for the lower line, $k=9.4, p=4.1$ for the upper one, in appropriate units. 
the profiles resemble the water profiles in other porous materials, determined by measuring the absorption of gamma-rays, see e.g. [9].

As the input data we have taken the well-seen water front at a large $z$, i.e. $z_{2}$, and the less clearly seen kink at which the plot starts to decline, $z_{1}$. Besides, the amplitude at $z=0$ has been taken as $m_{0}=0.30$, but this is only a matter of scale. Having only two meaningful numbers, in order to apply the above model, Eq. (7), we have to make some additional assumptions. After some numerical attempts, we had to assume $s=-5 / 4$, to reproduce shape of the experimental plot. Similarly we had to assume $r_{2} / r_{1}=100$, at least to the order of magnitude. Finally we have arrived at the model plots, which reproduce fairly well the experimental trends, see Fig. 4. Note that the ratio of calculated time parameters $k$ for these two plots is not far from the ratio of the experimental times of water flow in the brick. To summarise, the power law of decline for the $\rho(r)=r^{s-2}$ for $r_{1}<r<r_{2}$, zero otherwise, with $s=-5 / 4$ seems to mimic the real distribution function at least in its main features: there are plenty of narrow capillary tubes and a few broad ones. The range of radii is limited, and practically there is a cut of in the $\rho(r)$ function at small $r$. On assuming that the broadest tubes do not exceed $r=0.1 \mathrm{~mm}$, we can judge that the smallest radius appearing in large abundance should be about $0.001 \mathrm{~mm}$. This is as much as one can gain at present with the preliminary experimental data and simplifications allowed in the model fit procedure.

The present paper presents the results of first stages of a regular study of the liquid transport in porous materials at the NGR station at the IAE, Swierk.

\section{Acknowledgments}

The Authors are thankful to Dr Marton Balasko of KFKI, Budapest, for his effective expertise and help in creating the Neutron and Gamma Radiography Station at the IAE, Świerk. We appreciate the work of Ludwik Dąbkowski, M.Sc., and his colleagues from the Research Reactor Division of this Institute, on design and setting the mechanical equipment of the Station. An illuminating discussion on the liquid transport in porous materials with Professor J.A. Pogorzelski of the Building Research Institute, Warsaw, is thankfully appreciated.

\section{References}

[1] C.A. Black, Methods of Soil Analysis, Part 1, Am. Inst. Agronomy, Madison 1965.

[2] L. Pel, K. Kopinga, G. Bertram, G. Lang, J. Phys. D., Appl. Phys. 4, 675 (1995).

[3] H. Pleinert, H. Sadouki, F.H. Wittmann, Mater. Struct. 31, 224 (1998).

[4] D. Quendard, H. Sallee, Mater. Res. Soc. Symp. Proc. 137, 165 (1989).

[5] J.A. Pogorzelski, Building Thermal Physics, PWN, Warszawa 1976 (in Polish).

[6] T. Nemec, J. Rant, M. Kaling, in: Proc. IAEA Technical Committee Meeting on Neutron Beam Research, September 1999, Lisbon, Ed. F.G. Carvalho, Nuclear and Technological Institute, Lisbon 1999, p. 119. 
[7] F. Korosi, M. Balasko, E. Swab, Nondestr. Test. Eval. 16, 309 (2001).

[8] A. Czachor, J.J. Milczarek, Annual Report 2000-ISSN 1507-5478, IAE, Świerk 2000 .

[9] M. Leśniewska, J.A. Pogorzelski, Archiwum Inynierii Ladowej 27, 123 (1981) (in Polish).

[10] J.R. Philip, Adv. Hydrosci. 5, 215 (1965).

[11] C. Hall, Build. Environ. 12, 117 (1977). 